\title{
Reflets
}

Revue ontaroise d'intervention sociale et communautaire

\section{La violence faite aux femmes : analyse de la situation en Ontario français}

\section{Colette Godin et Ghislaine Sirois}

Volume 2, numéro 1, printemps 1996

Contrer la violence subie par les femmes et les enfants en milieu familial

URI : https://id.erudit.org/iderudit/026109ar

DOI : https://doi.org/10.7202/026109ar

Aller au sommaire du numéro

Éditeur(s)

Reflets : Revue ontaroise d'intervention sociale et communautaire

ISSN

1203-4576 (imprimé)

1712-8498 (numérique)

Découvrir la revue

Citer cet article

Godin, C. \& Sirois, G. (1996). La violence faite aux femmes : analyse de la situation en Ontario français. Reflets, 2(1), 116-133.

https://doi.org/10.7202/026109ar

Tous droits réservés (C) Reflets : Revue ontaroise d'intervention sociale et communautaire, 1996
Ce document est protégé par la loi sur le droit d'auteur. L'utilisation des services d'Érudit (y compris la reproduction) est assujettie à sa politique d'utilisation que vous pouvez consulter en ligne.

https://apropos.erudit.org/fr/usagers/politique-dutilisation/ 


\section{La violence faite aux femmes:}

\section{analyse de la situation en Ontario français}

\section{C olette $\mathbf{G}$ odin et $\mathbf{G}$ hislaine Sirois}

pour A ction ontarienne contre la violence faite aux femmes

\section{Introduction}

D ans cet article, nous avons réfléchi à une situation plus grande que le labeur quotidien. $N$ ous espérons, par cet exercice, nour rir la passion de l'action, alors que le gouvernement conservateur prend d'assaut les services sociaux et communautaires. Cette réflexion a eu lieu en octobre dernier. Elle a été entreprise par des femmes très actives et provenant des quatre coins de la province: Denyse Boulanger-C ulligan (Thunder Bay), Suzanne C ubaynes (C hatham), G aëtanne Pharand (Sudbury), Andrée Côté (Toronto), Colette Godin et Ghislaine Sirois (O ttawa). $C$ 'est de la violence faite aux femmes, plus précisément de la violence que vivent les femmes d'expression française de I'O ntario; de la présence, ou plutôt de l'absence, des services en français; des coupures budgétaires qui ont trouvé en $\mathrm{H}$ arris le messager et de la riposte des femmes, dont elles discutent. U ne réflexion ancrée dansl'action de cesfemmes, intervenantes, avocates, formatrices. 


\section{De la réalité francophone}

En 1993, la Fédération nationale des femmes canadiennesfrançaises, se présentant devant le Comité canadien sur la violence faite aux femmes, dressait ce portrait de la réalité des femmes francophones vivant en milieu minoritaire:

$D$ ans l'ensemble, la collectivité canadienne-française ne reconnaît pas complètement l'existence de la violence à l'égard des femmes, c'est une question sur laquelle les communautés ferment encore les yeux. Les valeurs traditionnelles qui prévalent dans les communautés isolées, et l'emprise de la religion catholique emmurent les femmes victimes de violence dans le plus grand silence, et dans l'acceptation de leur sort. La violence fait partie du domaine du privé.Toute émancipation est vue comme un danger, car dans les petites communautés francophones, elle signifie le démantèlement du modèle patriarcal.Tout comme dans la société canadienne, la violence contre les femmes en milieu minoritaire est un problème lié à la non-reconnaissance de l'égalité des sexes et, dans certains milieux, cette violence est doublée d'un problème liéàl'oppression du groupe lui-même. Lesfemmes francophones sont ainsi appelées à vivre un triple isolement: en tant que femmes, en tant que francophones, et, en tant que victimes de violence.

Au cours desannées, les femmes des groupes communautaires de l'O ntario ont tenté de rendre visible la violence faite aux femmes et, de briser le silence par des colloques sur les agressions à caractère sexuel ou sur l'intervention féministe, des rencontres, des festivals de vidéos, des kiosques dans les centres d'achats, et par leur présence auprès des médias. $M$ algré ces efforts, il n'en demeure pas moins que les femmes francophones attendent longtemps pour demander de l'aide. C ela est, en partie, dû à leur héritage traditionnel, par désir de préserver le couple, parce qu'elles sont «néespour un petit pain», parce qu'ellesse croient responsables de ce qui leur arrive. M ais, la fragmentation et les lacunes du réseau de services en français jouent aussi un rôle important dans 
I'utilisation limitée que font les femmes d'expression française des services en place.

\section{Services en français}

Au cours des dernières années, le gouvernement a subventionné certaines activités de sensibilisation et de prévention, et quelques groupes de femmes en ont fait leurs dossiers prioritaires. Citons, par exemple, la pièce de théâtre Interdit, produite par I'U nion culturelle des Franco- 0 ntariennes, une pièce de théâtre sur I'inceste qui a fait la tournée des écoles francophones de la province. La Fédération des femmes canadiennes françaises de I'O ntario, avec son outil de sensibilisation $L$ a violence démasquée, en est un autre exemple. $M$ ais, en $O$ ntario, les services de prévention et de sensibilisation en français pour les femmes victimes de violence sont encore trop peu nombreux et insuffisamment financés.

Si on s'est éloigné du financement de projets à court terme, qui n'assurait aucune continuité dans les services, c'est que depuis deux ans, le gouvernement de l'O ntario s'est engagé au développement de services en français dans les centres d'aide et de lutte contre les agressions à caractère sexuel. Pourtant, le nombre d'intervenantes est largement insuffisant. Par exemple, à Sudbury, pour mettre sur pied un centre ayant les ressources financières permettant l'embauche de deux intervenantes, et la mise en oeuvre d'une ligne opérant 24 heures par jour, sans oublier la recherche de financement, la formation, le recrutement de bénévoles, la présence dans la communauté, etc., relève du miracle, et constitue une recette parfaite de burnout.

II y a actuellement quatorze centres contre le viol qui offrent, ou sont en voie d'offrir, avec des ressources inadéquates, des services en français dans toute la province: centres francophones à $\mathrm{O}$ ttawa, Toronto, et Sudbury; des services bilingues à l'intérieur de centres existants à Pembroke, Timmins, Cornwall, 
W indsor, Sarnia, $N$ orth $B$ ay et dans les régions d'A Igoma et du $\mathrm{N}$ iagara et des points de services à $\mathrm{H}$ amilton et Barrie. $\grave{A}$ Thunder Bay, le projet tarde à être financé. Finalement la ligne téléphonique d'écoute et d'information SO S F emmes offre du soutien en tout temps, mais le service est menacé par les coupures.

II n'y a que cinq maisons d'hébergement qui soient en mesure d'accueillir dans leur langue les femmes d'expression française.

À $O$ ttawa, la $M$ aison d'A mitié a dû refuser plus de cinq cents femmes I'an dernier. À H awkesbury, une bonne partie de la clientèle provient du $\mathrm{N}$ ord, et les femmes violentées doivent se déplacer lorsque les maisons de M atheson ou de K apuskasing ne peuvent les accueillir pour des raisons d'éloignement ou de sécurité. Pour leur part, lesfemmes d'A lgoma quittent la province pour se réfugier dans les maisons du Q uébec.

À part ces services, nous trouvons des services d'aide dans les centres de ressourcescommunautaires, les agences de counselling, enfin dans le système de santé (hôpitaux, cliniques médicales). Toutefois, les femmes d'expression française doivent souvent frapper à plus d'une porte dans l'espoir, souvent vain, de se faire servir dans leur langue.

Pour les femmes immigrantes d'expression française les contraintes sont encore plus grandes: discrimination, racisme, précarité et vulnérabilité inhérentesà la condition de «parrainées». B ref, la ter re d'accueil n'est pas toujours accueillante. Lorsqu'elles portent plainte contre un mari violent, elles se retrouvent coincées par les règles de parrainage ou leur statut de réfugiées, souvent incomprises dans les services. Leur isolement est presque total. Le seul organisme destiné aux femmes noires d'expression française, et dirigé par elles, le R éseau des femmes noires francophones deT oronto, a fermé ses portes à l'été 1995.

Depuis l'arrivée au pouvoir du gouvernement conservateur en juin 1995, les couperets tombent. C ertains services en français sont touchés maisl'impact est plus sévère, car il menace l'existence même des services, comme on l'a vu pour les centres de santé communautaires de $\mathrm{H}$ amilton et de Welland. 
Le réseau développé par les femmes d'expression anglaise, qui a bénéficié du financement gouvernemental pendant des années, est lui aussi vulnérable. M ais, les services en français étant à l'état quasi embryonnaire, l'impact de ces mêmes coupures sur le réseau francophone sera encore plus dévastateur.

\section{Coupures réactionnaires Harris}

Les coupures que le gouvernement conservateur de l'O ntario impose à la population affectent particulièrement les femmes et les rendent plus vulnérables. En matière de violence faite aux femmes, mentionnons les coupures suivantes.

Les maisons d'hébergement ont subi des compressions de budget global, de plusl'0 utreach, les programmes de sensibilisation et de prévention sont éliminés. Les femmes auront moins accès à ces services qu'auparavant, et il sera plus difficile d'en connaître l'existence. De plus, intervenir en l'absence de programmes de prévention condamne une agence à «soigner les bobos» sans jamais s'attaquer à la cause du problème. Les compressions budgétaires touchent aussi les services d'interprétation, l'intervention directe auprès des femmes et des enfants, et la formation du personnel.

Les maisons de deuxième étape (second stage housing) ne seront plusfinancées. Lesfemmesqui ont besoin de soutien d'une durée de plus de six semaines, ou celles dont l'agresseur persiste à les harceler, n'auront plus accès à ce lieu sécuritaire et devront se réfugier dans les services anglophones.

$D$ ans plusieurs régions, ce sont les services de counselling au sein d'agences de services à la famille par exemple, qui se chargeaient des initiatives à l'intention des femmes violentées (groupe de soutien aux femmes qui subissent de la violence de leur partenaire, groupes de survivantes d'inceste, etc). Les coupures subies sont importantes, et menacent l'existence de certains de ces programmes. 
C oupures à SO S F emmes, la seule ligne provinciale en français qui offre écoute et soutien vingt-quatre heures par jour.

Les programmes à l'intention des hommes violents sont éliminés, sauf s'ils sont subventionnés par les Services correctionnels.

Par ailleurs, les coupures à l'aide sociale ( $21 \%$ des prestations) affectent en majorité les femmes. $\mathrm{Ce}$ sont elles qui ont habituellement la garde des enfants. Elles ont souvent de la difficulté à trouver un travail qui offre un revenu adéquat. II est aussi rare qu'elles disposent des avantages sociaux qui pallient à leur manque à gagner lorsqu'elles tombent malades. Q uitter une relation violente pour vivre et faire vivre ses enfants dans la pauvreté peut alors s'avérer impensable.

Les coupures à l'aide juridique, particulièrement en matière de droit de la famille (divorce, garde des enfants, etc.), affecteront surtout lesfemmes. Les coupures dans les services de garde auront aussi un effet sérieux sur la qualité de vie des femmes. En effet, les femmes incapables de se trouver un service de garde accrédité et public, quitteront leur emploi ou feront appel à des gardiennes du secteur privé, qui travailleront de 7 h 00 à 5 h 00 , sans avantages sociaux, afin de fournir repas et activités à bas salaire.

Les réformes relativesau workfare, prévues par le gouvernement, forceront les prestataires de l'aide sociale à accepter n'importe quel travail, à des conditions salariales inadéquates, sous peine de perdre leurs prestations A lors que les femmes qui ont des enfants de moins de trois ans seront exemptées, que feront celles qui ont des enfants de plus de trois ans en l'absence de garderies accessibles? D e plus, lorsqu'on sait que les billets d'autobus sont parfois un luxe que plusieurs ne peuvent s'offrir, comment en plus payer une gardienne? Finalement, les coupures au soutien à domicile restreignent l'autonomie des femmes handicapées, et ajoutent à la tâche de celles qui s'occuperont des personnes malades.

Toutes ces mesures portent atteinte aux droits, à la dignité et à I'autonomie des femmes. Elles mettent en péril leur santé et détériorent leurs conditions de vie.

Le gouvernement provincial n'est pasle seul responsable de la 
détérioration des conditions de vie des femmes. Au niveau fédéral, leschangements prévusà la politique d'assurance-chômage, la décision T hibaudeau concernant la taxation des pensions alimentaires et le désengagement en matière de santé et de sécurité sociale sont des exemples de mesures qui augmentent le désavantage social et économique des femmes. A u niveau juridique, dans les causes de violence sexuelle, les gains importants des femmes, obtenus à la suite des réformes du Code criminel, en 1983 et 1992 , ont vite été érodés. $D$ ans un article publié dans $F$ emmes d'A đion, Andrée Côté (1994) identifie trois stratégies utilisées par les avocats de la défense pour augmenter la gamme d'excuses qui sont disponibles aux hommes violents:

- la première consiste à discréditer le témoignage des femmes en invoquant le «syndrome de la fausse mémoire», en vertu duquel une femme pourrait être incitée par son ou sa thérapeute à reconstituer une fausse version des rapports entre elle et son agresseur présumé;

- la deuxième consiste à excuser le contrevenant en plaidant «qu'il souffrait d'une incapacité causée par un état avancé d'ébriété» invoqué dans l'affaire $D$ aviault;

- enfin, la troisième stratégie consiste à perquisitionner les dossiers privés et confidentiels des victimes afin de trouver des indices permettant de discréditer leur témoignages.

$\mathrm{Si}$, à la suite de la décision de la C our Suprême, le gouvernement fédéral est intervenu pour limiter l'usage de la défense pour ébriété, il ne manifeste pas le même empressement à protéger le droit à la confidentialité desfemmes après que la C our Suprême ait accordé aux avocats de la défense l'accès aux dossiers confidentiels des femmes, à la demande des juges. Le message pernicieux du soidisant syndrome de la fausse mémoire fait son chemin en procurant une chance de plus à l'agresseur d'obtenir l'impunité.

Pour ceux et celles qui ne croiraient pas encore à un backlash contre les femmes, ajoutons la montée du fondamentalisme religieux. Près de nous, on commet des attentats meurtriers pour détruire les cliniques d'avortement. Ce fondamentalisme se manifeste aussi par le refus obstiné de l'Église catholique de reconnaître la pleine égalité des femmes. 
C e backlash est une réaction aux victoires remportées par lesfemmes au cours des trente dernières années, et il se manifeste dans les politiques gouvernementales et les attitudes sociales. II vise à ramener les femmes «à leur place», c'est à dire à la maison. Pourtant, nousle savons, la maison n'est pas toujours un lieu sécuritaire pour les femmes.

«a violence contre les femmes constitue une grave atteinte aux droits de la personne, notamment des droits garantisàl'article 7 de la $C$ harte canadienne des droits et libertés, à savoir le droit à la vie, à la liberté et à la sécurité de sa personne» (C ôté, 1995).

Dans une société qui s'est dotée de garanties constitutionnelles visant à assurer le plein respect des droits à l'égalité des femmes, nous serions en mesure de nous attendre à ce que l'État en assure le respect. Les gouvernements doivent faire en sorte que leurs politiques n'augmentent pas le désavantage économique et social des femmes, ce que semblent ignorer les partisans de la vague néo-libérale qui déferle sur le pays. La violence faite aux femmes ne diminuera que lorsque diminueront les inégal ités entre hommes et femmes.

\section{Riposte des femmes}

D ans la conjoncture actuelle, les femmes francophones consacrent toute leur énergie à préserver les acquis. $C$ 'est là certainement le rôle qu'assure A dion ontarienne contre la violence faite aux femmes (AO CVF, organisme créé en 1988). AO CVF vise à soutenir le développement de services en français, diffuse l'information concernant la violence faite aux femmes, fait des démarches politiques, produit du matériel de formation, etc. AO CVF sert de point de rencontre aux femmes impliquées dans les services en vue $d$ 'analyser des enjeux qui les concernent.

LaTable féministe francophone de concertation provinciale de l'O ntario (TFFCPO) est aussi porteuse d'un mandat de concertation: recherches sur la santé et les droits des femmes, 
développement d'outils de communication, formation sur les droits à l'égalité, élaboration de projets concernant l'autonomie financière des femmes. Le maintien de ces lieux de concertation est essentiel à la solidarité de la communauté des femmes francophones.

A lors que le gouvernement se désengage de ses responsabilités, le financement d'activités représente un défi de taille tant pour la concertation que pour les services directs. Certains groupes envisagent des projets d'auto-financement. C ette solution comporte l'avantage pour les groupes de ne plus dépendre pour leur survie de subventionsaléatoires. Par contre, l'énergie, le temps, les ressources humaines nécessaires aux appels de fonds ne sont pas à la portée de la majorité des groupes communautaires. N ous devons donc continuer à exiger des gouvernements qu'ils s'acquittent de leurs obligations à l'égard des femmes, et en particulier, celles qui sont victimes de violence. Les femmes ont droit à l'équité et à la sécurité en tant que citoyennes, et elles ont aussi droit aux fonds publics nécessaires pour se les assurer.

C'est pour rappeler au gouvernement de l'O ntario ses obligations àl'égard de droits des femmes, que la TFFCPO et AO CVF ont rédigé la $D$ éclaration des femmes de l' 0 ntario (1995). Présentée au gouvernement le 6 décembre 1995, et endossée à cette date par plus de 150 groupes de femmes, syndicats, groupes populaires et autres, tant francophones que d'expression anglaise, la D éclaration exige un moratoire immédiat sur toutes les coupures affectant les femmes: violence faite aux femmes, garderies, logement, aide sociale, droit du travail, aide juridique, etc. De plus, au nom des femmes, elle demande au gouvernement de changer radical ement sa façon d'administrer la chose publique, en respectant les principes d'égalité, d'équité, de légal ité et d'universalité. Pourtant, dans les régions, les femmes tentent d'obtenir d'autres signatures et à faire connaître à leurs députés leur mécontentement. En guise de conclusion, nous présentons ici, avec l'accord des groupes qui l'ont produit, le texte intégral de la D éclaration, dans l'espoir que vous vous joindrez à la lutte de milliers d'autres $O$ ntarienness et $O$ ntariens. D ans cette $D$ éclaration, la lectrice ou 
le lecteur constatera que l'on exigeait une réponse du gouvernement $\mathrm{H}$ arris pour le 8 mars 1996, date de la Journée internationale des femmes. 0 r, à cette même date, personne n'a daigné répondre à cet appel.

$M$ algré les menaces de représailles, formulées par divers représentants et représentantes de ce gouvernement, il n'est pas encore temps de se taire. II faut plutôt clamer bien haut que «les femmesne sont pas nées pour se soumettre» surtout pas lesFrancoO ntariennes, dont l'histoire le confirme...

\section{Déclaration des femmes de 1'Ontario}

\section{le 6 décembre 1995}

$\mathrm{N}$ ous, les femmes de l'O ntario, avons décidé de nous rassembler pour déclarer notre opposition aux politiques actuelles du gouvernement de I'O ntario, et pour exiger un changement radical dans sa façon de gouverner la province.

Attendu que le gouvernement de $\mathrm{M}$ ike $\mathrm{H}$ arris vient d'annoncer une réduction vertigineuse de 8 milliards de dollars de dépenses, notamment 800 millions dansl'éducation, 1,3 milliarda dansles services hospitaliers et 1,4 milliards dans les transferts aux municipalités, ce qui représente $47 \%$ des transferts dans les deux prochaines années; et attendu que ce même gouvernement a pris des mesures suivantes depuis qu'il est au pouvoir:

- réduction de $21,6 \%$ des sommes allouées aux bénéficiaires de l'aide sociale;

- élimination des fonds de soutien pour de nouvelles garderies sans but lucratif;

- coupures dans les services de transport pour les personnes handicapées;

- élimination du financement destiné à la prévention de la violence conjugale;

- élimination du financement des services d'hébergement de deuxième étape pour les femmes victimes de violence conjugale; coupures dans le financement des autres services d'hébergement; 
- coupures dans les programmes d'accueil destinés à faciliter l'établissement de nouveaux immigrants;

- coupures majeures dans les subventions aux arts et à la culture;

- élimination du financement de nouvelles cliniques d'avortement;

- coupures dans les services de counselling familial;

- élimination de subventions à la ligne d'écoute téléphonique pour la jeunesse gaie et lesbienne;

- coupures dans les fonds alloués à la protection de la jeunesse, et réduction des services offerts aux survivantes d'inceste;

- réduction du financement des services sociaux adaptés aux besoins spécifiques des minorités culturelles;

- coupures dansles services en français offerts par lesmunicipalités et dans plusieurs ministères; coupures dans les services sociaux offerts en français dans plusieurs villes, dontToronto, $\mathrm{H}$ amilton et Welland;

- adoption de nouveaux règlements qui pénalisent les assistées sociales si elles partagent leur domicile avec un homme;

- abolition de la Loi sur l'équité en emploi;

- gel des sommes disponibles pour l'équité salariale, et élimination de plus de 100000 femmes des bénéfices de l'équité salariale;

- abolition du programme de création d'emploi Boulot-O ntario;

- coupures radicales dans le financement des programmesd'habitations à loyer modique et des coopératives d'habitation et privatisation des logements sociaux;

- coupures dans les services offerts aux femmes enceintes;

- coupures dans le financement des services de santé dans les hôpitaux et les soins à domicile; coupure de 10 millions dans le programme qui fournit de l'oxygène à domicile aux malades;

- fermeture du W omen's H ospital;

- abolition du Bill 40, qui interdisait notamment le recours aux briseurs de grève; introduction d'une législation mettant en péril d'importants acquis syndicaux;

- mise en place d'une ligne téléphone de délation contre les assistées sociales;

- abolition de la gratuité des médicaments pour 380000 personnes âgées et 1,3 millions de bénéficiaires de l'aide sociale 
en imposant un ticket modérateur de 2 \$ pour chaque médicament; de plus, on permet aux pharmaciens d'exiger à la majorité des personnes âgées des «frais de service» de 6,11 \$ pour chaque ordonnance, en plus de leur imposer des sfrais d'usager» de 100 \$ par année;

- autorisation donnée aux municipalités de facturer des services comme le ramassage des ordures, l'accès aux parcs publics et aux bibliothèques;

- autorisation donnée aux collèges communautaires et aux universités de hausser les frais de scolarité de 15 et $20 \%$ respectivement;

- soutien à la privatisation de services publics par les municipalités;

- hausse des taxes scolaires et des impôts fonciers encouragée par le gouvernement.

Attendu que le gouvernement $\mathrm{H}$ arris annonce qu'il a l'intention de prendre les mesures suivantes dans un avenir rapproché:

- démantèlement de la législation relative au contrôle des loyers;

- introduction du workfare;

- coupures dans les fonds alloués à l'aide juridique;

- élimination de cliniques spécialisées d'aide juridique comme le C entre d'aide juridique pour l'équité salariale;

- abolition de laA dvocacy C ommission et abrogation de la A dvocacy A ct;

- amendement du L abour Standards A ct pour limiter les réclamations qui pourront être mises de l'avant par les employées qui auront perdu leur emploi à la suite de la fermeture de leur entreprise;

- affaiblissement des règlements visant la protection de l'environnement;

- coupures dans les mécanismes de contrôle de la santé et de la sécurité des travailleuses, notamment les services d'enquêtes sur les conditions de travail dangereuses;

- abolition des subventions pour 68000 places en garderie;

- redéfinition de la notion de handicap, et élimination éventuelle du soutien financier accordé à des milliers de personnes handicapées; 
ATTENDU que le gouvernement ne reconnait pas le rôle des groupes de femmes dans le processus démocratique, et qu'il menace de couper les subventions aux groupes qui critiquent ses politiques, faisant ainsi preuve de la même attitude que certains hommes qui tentent de contrôler leur conjointe par les menaces et la violence.

A TTEN Du que le gouvernement $H$ arriss'aligne sur les intérêts de la finance internationale et des multinationales en réduisant l'intervention de l'État à des mesures qui ne profiteront qu'aux classes aisées, et qu'il s'est déjà engagé à adopter les mesures suivantes:

- abolition desfrais de déclaration annuelle descompagnies(filing fee), au coût de 15 millions pour le trésor public;

- réduction de $30 \%$ des impôts sur le revenu, dans les trois prochaines années, ce qui représente un manque à gagner de 5 milliards pour le gouvernement.

ATTENDU que les effets immédiats et futurs de ces mesures seront les suivants:

- accroissement de la pauvreté des femmes les plus vulnérables;

- accroissement de la pauvreté des enfants alors qu'àToronto, un enfant sur trois vit actuellement de l'aide sociale;

- dégradation de la santé et de la sécurité physique desfemmes;

- dégradation de conditions de vie de la population;

- détérioration des conditions de logement, et multiplication des sans abris;

- perte d'autonomie pour les personnes handicapées;

- maintien de barrières qui excluent les femmes, et plus particulièrement les femmes de couleur, autochtones et handicapées du marché du travail; ou qui les maintiennent dans des ghettos d'emploi sans chance de promotion;

- augmentation de la violence conjugale;

- embrigadement des femmes dans la dépendance à l'égard de leur conjoint;

- augmentation du chômage, avec une prévision de 4300 de pertes d'emploi directes et 120000 pertes d'emploisindirectes,

- détérioration des conditions de travail des femmes;

- augmentation de la vulnérabilité des travailleuses face au harcèlement sexuel et racial; 
- érosion des droits acquis des francophones à des services en français;

- érosion des droits des minorités culturelles et ethniques à des services sociaux et de santé adaptés à leurs besoins;

- dégradation accrue de l'environnement;

- augmentation de la charge de travail des femmesà la suite des coupures dans les services sociaux et de santé;

- érosion du droit de toutes les femmes de participer également au processus démocratique de la société;

- dégradation de la solidarité sociale;

- aggravation de la vulnérabilité, de la dépendance et de l'oppression des femmes.

Force nous est de constater que le gouvernement $\mathrm{H}$ ar ris se livre à une véritable guerre contre les femmes.

Nous jugeons que les politiques mises en oeuvre par le gouvernement violent les droits fondamentaux des femmes garantis par la C harte canadienne des droits et libertés et par le droit international du droit de la personne. Par exemple:

- les coupures à l'aide sociale portent atteinte au droit à «un niveau de vie suffisant, y comprisune nour riture, un vêtement et un logement suffisant, ainsi qu'à une amélioration constante dans ses conditions d'existence» garanti dans le Pacte international relatif aux droits économiques, sociaux et culturels (article 11).

- I'introduction du workfare porterait atteinte au droit à un travail «ibrement choisi ou accepté et porterait atteinte au droit à des conditions de travail justes et favorables», «un salaire équitable et une rémunération égale pour un travail de valeur égale sans distinction aucune» qui sont garantis dans le Pacte international relatif aux droits économiques, sociaux et culturels (articles 6 et 7).

- les coupures dans les programmes de soutien aux femmes violentées et les programmes de prévention contre la violence conjugale mettent en danger la vie des femmes, leur sécurité physique et psychologique et leur liberté, garantis par la Charte canadienne des droits et libertés (article 7). 
Elles sont contraires à l'obligation d'élaborer des approches fondées sur la prévention, qui favorisent la protection des femmes contre toutes formes de violence et qui assurent la mise en place de services spécialisés, énoncée dans la D éclaration sur l'élimination de la violence des femmes, adoptée en février 1994 par l'A ssemblée générale de l'o nu (article 4).

Lorsque le gouvernement viole ses obligations constitutionnelles et internationales àl'égard des droits des femmes, il outrepasse les paramètres de la légalité et il agit de façon illégale. Ce faisant, le gouvernement $\mathrm{H}$ arris a perdu toute légitimité à nos yeux.

En ce 6 décembre 1995, nous, les femmes de l'O ntario, déclarons que nous nous engageons à défendre les droits fondamentaux de nos mères, de nos soeurs, de nos filles, de nos amies, nos amantes et nos voisines. N ous défendrons les acquis juridiques, économiques, sociaux et politiques que les femmes ont gagnés par de haute lutte au cours du $X X{ }^{\mathrm{e}}$ siècle.

II n'est pas question de retourner en arrière, et comme les milliers de femmes réunies à Beijing, en septembre dernier, le chantaient: T here is no going back!

Par la présente, nous mettons en demeure le gouvernement $\mathrm{H}$ arr is de cesser immédiatement ses politiques de coupures; elles sont discriminatoires et elles mettent en péril le réseau de soutien et de services que lesfemmes ont mis sur pied au cours des trente dernières années.

$\mathrm{N}$ ous demandons que le gouvernement change radicalement d'approche dans son administration de la chose publique. N ous le sommons de gouverner de façon égalitaire et équitable, et de s'abstenir d'exercer son pouvoir uniquement en fonction des intérêts de la haute finance et des hommes d'affaire.

- Son administration doit être égalitaire, c'est-à- dire qu'elle doit tendre à réduire, et non pas aggraver, les désavantages sociaux et économiques des femmes et des hommes des groupes historiquement défavorisés;

- son administration doit être équitable, c'est-à-dire qu'elle doit accorder le même bénéfice et la même protection aux femmes et aux hommes de toute couleur, origine ethnique ou culturelle, 
orientation sexuelle et condition sociale, tant au niveau des objectifs de ses politiques et programmes, que dans leurs modalités d'application;

- son administration doit être légale et doit respecter les principes universels de la justice fondamentale. Elle doit notamment, respecter la dignité et la liberté humaine, ainsi que la légalité et l'indépendance des femmes;

- le bénéfice de son administration doit être universel, concrètement et efficacement livré à toutes les personnes vivant sur le territoire de la province.

N ous exigeons que le gouvernement décrète un moratoire immédiat sur toute coupure ou réduction de services susceptibles d'aggraver les désavantages que subissent les femmes.

N ous exigeons que d'ici le 8 mars 1996, Journée inter nationale des femmes, le gouvernement nous dise quelles mesures concrètes il entend prendre pour promouvoir les droits des femmes et pour rétablir, sauvegarder, renforcer et promouvoir nosdroits ainsi que les lois, les programmes et les services suivants:

- un réseau public de garderies;

- l'accès à un logement convenable et le contrôle des loyers;

- le droit à un travail et de jouir de conditions de travail justes et favorables;

- l'équité salariale;

- un transport public, en ville et en région;

- le droit à la santé;

- desmesures contre la violence conjugale et la violence sexuelle;

- la sécurité du revenu;

- les droits linguistiques et des services en français;

- l'aide juridique;

- le rétablissement du Bill 40 et la reconnaissance pleine et entière du droit à la syndicalisation;

- la Loi sur l'équité en emploi;

- les droits des peuples autochtones;

- les services sociaux et de santé adaptés aux besoins spécifiques des communautés minoritaires;

- la citoyenneté des femmes immigrantes; 
- la santé et sécurité au travail;

- le droit à l'éducation.

$\mathrm{N}$ ous attendons la réponse du gouvernement $\mathrm{H}$ arris le 8 mars prochain.

$\mathrm{N}$ ous, les femmes de l'O ntario, estimons qu'il s'agit d'un état d'urgence. N ous avisons formellement le gouvernement de I'O ntario que s'il continue de s'attaquer aux droits des femmes, nous prendrons toutes les mesures nécessaires pour assurer la légitime défense de nos droits. Les groupes de femmesne plieront pas devant les menaces et la censure du gouvernement.

\section{Bibliographie}

CÔTÉ Andrée (1994). La guerre des sexes n'est pas finie, Femmes d'Action, vol. 24, no 1, 24-33.

CÔ TÉ Andrée, (1995). La violence contre les femmes et la réforme du droit criminel, $R$ ecommandations en vue d'une réforme égalitaire du droit criminel. 0 ttawa, Action 0 ntarienne contre la violence faite aux femmes.

COMITÉ RÉSEAU (mai 1993). Forum sur les "Services d'approches» pour les femmes francophones, rapport synthèse $O$ ttawa, comité $R$ éseau.

CO M ITÉ R ÉSEAU (avril 1994). F orum sur les «Services d'approches» auprès des femmes immigrantes et de minorités radales, rapport synthèse. O ttawa, C omité $R$ éseau.

CO M ITÉ R ÉSEAU (juillet 1994). Y a-t-il des besoins de connaissances sur la question faite aux femmes francophones dans la région d' $O$ ttawa- $C$ arleton, et si oui, lesquels? $O$ ttawa, $C$ omité $R$ éseau.

COMITÉ DES FRANCOPHONES DU NORD-OUEST DE L'ONTARIO et ACCUEIL FR AN C O PHONE DETHU NDER BAY (mai 1994). S'informer, c'est prévenir, É tude de besoins pour les femmes francophones du $\mathrm{N}$ ord-ouest de l' $\mathrm{O}$ ntario au sujet de la violence faite aux femmes et de I'agression à caradère sexuel. Thunder Bay, Comité des francophones du N ord-ouest de l'O ntario et Accueil francophone deT hunder Bay.

CON VER GEN CE (octobre 1992). R endre visible I'invisible, R apport sommaire de l'étude de besoins visant la création de services en français à l'intention des survivantes d'agression à caractère sexuel résidant dans la région de la $C$ apitale nationale, $O$ ttawa, $C$ oopérative $C$ onvergence.

COLLOQUE PROVINCIAL SUR LES AGRESSIONS À CARACTĖRE SEXUEL, C O LLECT IF (octobre 1994). Sensibiliser, déider, agir, L esA des du colloque provindal sur les agressions à caractère sexuel. $O$ ttawa, Colloque provincial sur les agressions à caractère sexuel.

CÔTÉ,AN DR ÉE ET A L (1993). «D ossier: la violence faite aux femmes», D roits et L ibertés, vol.16, no 1, juin 1993.

ONTAR IO ASSO CIATIO N O F INTERVALAN DTR AN SITIO N HOU SES (OAITH) (1995). "L et's take back the keys», A sodal action report, O ntario. 
FÉDÉR ATION N AT IO N ALE DES FEM M ES CAN ADIEN N ES-FR AN ÇAISES (1993). Situation des femmes en milieu minoritaire, mémoire présenté au $C$ omité canadien sur la violence faite aux femmes, 0 ttawa, Fédération nationale des femmes canadiennes-françaises.

Comité permanent de la santé et du bien- être social, des affaires sociales, du troisième âge, et de la condition féminine (1991). La guerre contre les femmes, $R$ apport du Comité permanent de la santé et du bien-être sodal, des affaires sociales, du troisième âge, et de la condition féminine, 0 ttawa, M inistère de l'approvisionnement et services.

FÉDÉR ATIO N N AT IO N ALE DES FEM M ES CAN ADIEN N ES-FR AN Ç AISES (1993). Situation des femmes en milieu minoritaire, M émoire présenté au CCSVF, 0 ttawa, Fédération nationale des femmes canadiennesfrançaises.

ACTION ONTARIEN NE CONTRE LAVIO LEN CE FAITE AUX FEM M ES (AOCVF) (1995). $D$ édaration des femmes del' $0 \mathrm{ntari}, 0$ ttawa,A ction ontarienne contre laviolence faite aux femmes. 